\title{
Assessment of the caudate nucleus and its relation to route learning in both congenital and late blind individuals
}

\author{
Patrice Voss ${ }^{1}$, Madeleine Fortin ${ }^{2}$, Vincent Corbo ${ }^{3}$, Jens C Pruessner ${ }^{3}$ and Franco Lepore $2^{2^{*}}$
}

\begin{abstract}
Background: In the absence of visual input, the question arises as to how complex spatial abilities develop and how the brain adapts to the absence of this modality. As such, the aim of the current study was to investigate the relationship between visual status and an important brain structure with a well established role in spatial cognition and navigation, the caudate nucleus. We conducted a volumetric analysis of the caudate nucleus in congenitally and late blind individuals, as well as in matched sighted control subjects.

Results: No differences in the volume of the structure were found either between congenitally blind (CB) and matched sighted controls or between late blind (LB) and matched sighted controls. Moreover, contrary to what was expected, no significant correlation was found between caudate volume and performance in a spatial navigation task. Finally, consistent with previously published reports, the volume of the caudate nucleus was found to be negatively correlated with age in the sighted; however such correlations were not significant in the blind groups.
\end{abstract}

Conclusion: Although there were no group differences, the absence of an age-volume correlation in the blind suggests that visual deprivation may still have an effect on the developmental changes that occur in the caudate nucleus.

Keywords: Blindness, Caudate nucleus, Spatial navigation, Volumetric MRI

\section{Background}

Spatial cognition and the ability to properly navigate in one's environment are believed to result from the contribution of several subcortical structures such as the hippocampus $(\mathrm{HC})$ and the caudate nucleus $(\mathrm{CN})$. This is well documented in rats, for instance, as place learning involves two different memory systems subserved by the $\mathrm{HC}$ and the dorsal striatum (particularly the caudate nucleus) [1-3]. In the early phases of learning, the $\mathrm{HC}$ is involved in the rapid acquisition of spatial information, allowing for rats to reach a target from any starting position [1]. The dorsal striatum is involved in a slower learning process [4] that relies on rewarded stimulus-response (S-R) behaviour $[3,5]$, i.e. gradually learning particular body turns in response to stimuli, which allow the rats to reach a target location from one starting point [6]. A similar segregation has been observed in humans. Functional

\footnotetext{
* Correspondence: franco.lepore@umontreal.ca

${ }^{2}$ Centre de Recherche en Neuropsychologie et Cognition (CERNEC),

Université de Montréal, Montréal, Canada

Full list of author information is available at the end of the article
}

Magnetic Resonance Imaging (fMRI) studies have shown that tasks requiring spatial representations preferentially activate the $\mathrm{HC}$, while tasks not requiring a particular 'spatial strategy activate mainly the $\mathrm{CN}[7,8]$. Moreover, gray matter density in these structures is found to correlate with specific navigational strategies [9]; subjects who were qualified as "spatial learners" had significantly more grey matter in the $\mathrm{HC}$ and less in the $\mathrm{CN}$ compared to "response learners".

Given the importance of vision and visual cues for spatial navigation and wayfinding, the absence of the visual modality raises questions on not only the ability to navigate in one's environment without vision but also on the anatomical and functional consequences to the brain structures involved in such learning. Despite the absence of visual inputs, blind individuals are nonetheless able to properly orient themselves and navigate in space $[10,11]$. To date, three studies have examined the effects of visual loss on the structural integrity of the HC [11-13]. While initial results may have seemed at odds with each other, with Fortin et al. [11] finding an increase in volume 
in the anterior portion of the $\mathrm{HC}$ and Chebat et al. [12] finding a decrease in volume in the posterior portion, a subsequent study by Lepore et al. [13] actually confirmed both sets of findings, suggesting that following blindness there is a shift of the neuronal population towards the anterior portions of the hippocampus compared to sighted individuals. Importantly, however, little is known about the consequences of visual loss on the structural anatomy of the $\mathrm{CN}$. Interestingly, in our previous study [11], where both blind and sighted subjects were asked to learn new paths in a human-size labyrinth (i.e. route learning task), most subjects anecdotally reported using a 'response learners' strategy where they attempted to sequentially recall the series of right and left turns that were required to properly follow the taught route. Indeed, previous work has not only shown the blind to possess superior serial memory for sequences [14], but that they also construct mental representations of paths via serial memorization of segmented inputs from each location along the path [15]. Moreover, crucial to our hypothesis formulation, recent data has in fact shown that route learning abilities in young and older adults are positively correlated to $\mathrm{CN}$ volume [16]. Therefore, given the above mentioned findings, we hypothesized here that blind individuals would not only possess larger $\mathrm{CN}$ relative to their sighted counterparts, but also that the volume would be significantly related to previously obtained behavioral measures in the blind [11]. To address this, using the MRI scans previously obtained in the aforementioned hippocampus volumetric study [11], we performed volume measurements of the $\mathrm{CN}$ in congenitally blind, late blind and matched sighted controls. We furthermore wanted to assess whether known associations with the caudate volume would also hold true in blindness. For instance, in normally-sighted individuals, the caudate volume is known to decrease with age $[17,18]$, and there are conflicting reports on how the caudate and hippocampal volumes covary with one another $[9,19,20]$. The latter investigation is especially pertinent to the present study, given the previously established relationship between hippocampal volume and route learning performance.

\section{Methods}

\section{Subjects}

Thirty participants, with no history of neurological, cognitive or sensory-motor deficits, other than blindness in the case of the blind participants, took part in the study and were divided into four groups (see Table 1 for demographic data on the blind participants). The first group $(n=8)$ consisted of congenitally blind $(C B)$ individuals (age: $31.1 \pm 9.5 \mathrm{y}$; gender: $6 \mathrm{~m} / 2 \mathrm{f} ; 7$ right-handers), while the second group $(n=8)$ were sighted $(\mathrm{SCB})$ participants (age: $31.4 \pm 9.4 \mathrm{y}$; gender: $6 \mathrm{~m} / 2 \mathrm{f} ; 7$ right-handers) paired for age, gender, education and laterality, as assessed by the Edinburgh Handedness Inventory [21]. The third group $(\mathrm{n}=7)$ consisted of late-blind (LB) individuals (age: $39.9 \pm 12.7$ y; gender: $4 \mathrm{~m} / 3 \mathrm{f}$; 6 right-handers) who all lost their vision after 14 years of age (age of onset: $21.28 \pm 6.55 \mathrm{y})$. The last group $(\mathrm{n}=7)$ were sighted participants (SLB) matched with the LB individuals (age: $40.3 \pm 13.4$ y; gender: $4 \mathrm{~m} / 3 \mathrm{f}$; 6 right-handers). The research protocol was approved by the ethics committees of the Center for Interdisciplinary Research in Rehabilitation, which coordinates in the Province of Quebec research

Table 1 Subject demographic information

\begin{tabular}{cccccll}
\hline Subjects & Age & Sexe & Onset & Duration & LP & Causes of blindness \\
\hline EB1 & 19 & M & 0 & 19 & Yes & Leber's congenital amaurosis \\
EB2 & 22 & M & 0 & 22 & No & Leber's congenital amaurosis \\
EB3 & 46 & F & 0 & 46 & Yes & Rubella \\
EB4 & 35 & M & 0 & No & Retinal detachment \\
EB5 & 39 & M & 0 & 35 & No & Retinitis pigmentosa \\
EB6 & 31 & M & 0 & 39 & No & Congenital glaucoma \\
EB7 & 35 & M & 0 & 31 & Yes & Leber's congenital amaurosis \\
EB8 & 22 & F & 0 & 35 & No & Retinal detachment \\
LB1 & 24 & F & 17 & 22 & Yes & Glaucoma \\
LB2 & 40 & M & 21 & 7 & No & Unknown \\
LB3 & 47 & F & 27 & 19 & Yes & Ischemic retinopathy \\
LB4 & 22 & M & 16 & 20 & Yes & Retinitis pigmentosa \\
LB5 & 57 & M & 33 & 6 & Yes & Medical accident (retina damage) \\
LB6 & 42 & M & 15 & 24 & No & Congenital cataracts and glaucoma \\
LB7 & 47 & F & 20 & 27 & No & Glaucoma and aniridia \\
\hline
\end{tabular}

The 'Onset' column refers to the age at which the subjects lost their sight. The 'Duration' column refers to the number of years that the subjects have been blind. The 'LP' column indicates whether subjects still had any residual light perception. 
with blind participants and is sponsored by the Institut Nazareth \& Louis Braille, by the Research Center of the Institut Universitaire de Gériatrie de Montréal, where the testing was carried out, by the Centre Hospitalier de l'Université de Montréal (CHUM), where the MRI scans were collected, and by the Université de Montréal, where the project originated. All participants provided written informed consent prior to testing.

\section{Image acquisition and analysis}

For each participant, MR images were previously acquired (see [11]) on a Siemens 1.5 Tesla Magnetom Vision MRI scanner (Siemens, Erlangen, Germany) at the Notre-Dame Hospital (CHUM). All images were acquired in high resolution $(1 \times 1 \times 1 \mathrm{~mm}, \mathrm{~T} 1$-weighted $3 \mathrm{D})$ with a sagittally oriented echo sequence (TR: 1100; TE:4.38; flip angle of $15 ; 256 \times 256$ matrix and FOV:250).

The raw structural files were first corrected for nonuniformities [22], and then registered into standard stereotaxic space based on the MNI 152 template [23]. This transformation results in an alignment along the AC-PC axis and accounts for individual differences in global brain size and shape. The images were then classified into different maps of gray matter, white matter, and cerebrospinal fluid (CSF) [24]. This procedure included the removal of all extracranial tissue, dura and the cerebellum. The resulting brain images were subsequently non-linearly registered and used to compute labels for the $\mathrm{CN}$ with the software ANIMAL [25], using each subject's classified maps. ANIMAL generates labels by multiplying the voxel values of the templates of the $\mathrm{CN}$ with each voxel's value across the individual grey matter maps (values of 1 for grey matter, 0 for either white matter or CSF). Manual corrections and volume assessments were performed by a trained rater (PV) to correct for gray-white matter boundaries and partial volume effects using the software package DISPLAY, developed at the Montreal Neurological Institute [26,27]. DISPLAY allows simultaneous viewing and navigating of brain volumes in coronal, sagittal, and horizontal orientations in $1 \mathrm{~mm}$ slice intervals. Figure 1 illustrates the resulting segemented caudate nucleus in one of the subjects.

Following the manual corrections, the reliability of the measurements was ascertained by obtaining estimates of both the intra-rater reliability and inter-rater reliability. The intra-rater intra-class reliability coefficients were 0.971 (left $\mathrm{CN}$ ) and 0.988 (right $\mathrm{CN}$ ), and were obtained by the original rater (PV) re-measuring the $\mathrm{CN}$ volume in four randomly selected MRI scans, with at least two months elapsing between consecutive measurements. The interrater intra-class reliability coefficients were 0.926 (left $\mathrm{CN}$ ) and 0.942 (right $\mathrm{CN}$ ), and were obtained by two raters (PV and MF) measuring caudate volumes independently in eight randomly selected MRI scans.

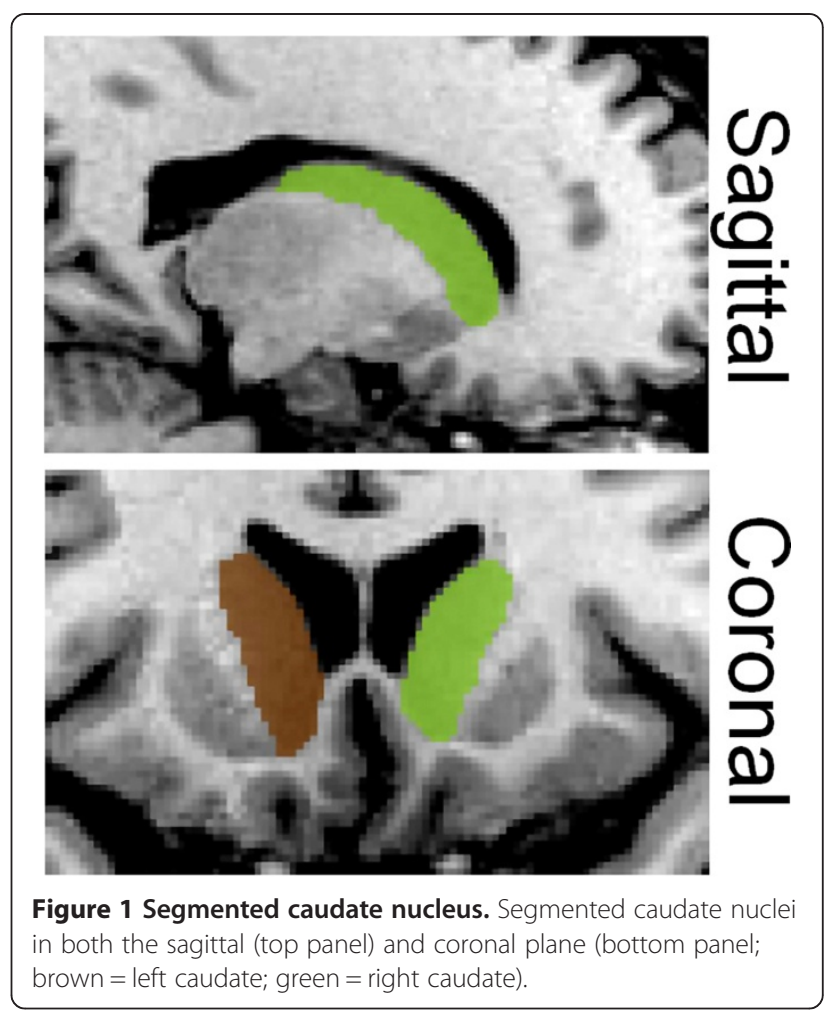

\section{Behavioral route learning task}

The task is described in greater detail Fortin et al. [11]. Briefly, the route learning task was performed as follows: subjects were asked to memorize a traveled route within a human-size labyrinth, guided by an experimenter, and then had to follow the same path alone on five subsequent trials while trying to make as few errors as possible; when mistakes were made, subjects were instructed to stop and were repositioned by the experimenter in the correct direction. Sighted subjects performed the task blindfolded. Four different routes were tested of increasing difficulty: with six, eight, ten and twelve decision points to memorize.

\section{Results}

\section{Volumetric analyses}

Separate analyses were performed for both blind groups since they were not matched with one another. A first two (group: $\mathrm{CB}$ and $\mathrm{SCB}$ ) by two (hemisphere) repeated measures ANOVA revealed that there was no significant group effect $(\mathrm{F}=0.034 ; \mathrm{p}=0.856)$, no effect of hemisphere $(\mathrm{F}=0.241 ; \mathrm{p}=0.631)$, nor a significant group $\mathrm{x}$ hemisphere interaction $(F=0.314 ; p=0.584)$. A second two (group: $L B$ and SLB) by two (hemisphere) repeated measures ANOVA did reveal a significant hemisphere effect $(F=6.995 ; \mathrm{p}=$ 0.021 ) as the right caudate was found to be larger than the left one (see Figure 2); however the group effect $(\mathrm{F}=2.659 ; \mathrm{p}=0.129)$ and the group $\mathrm{x}$ hemisphere interaction $(\mathrm{F}=0.465 ; \mathrm{p}=0.508)$ were non-significant. 


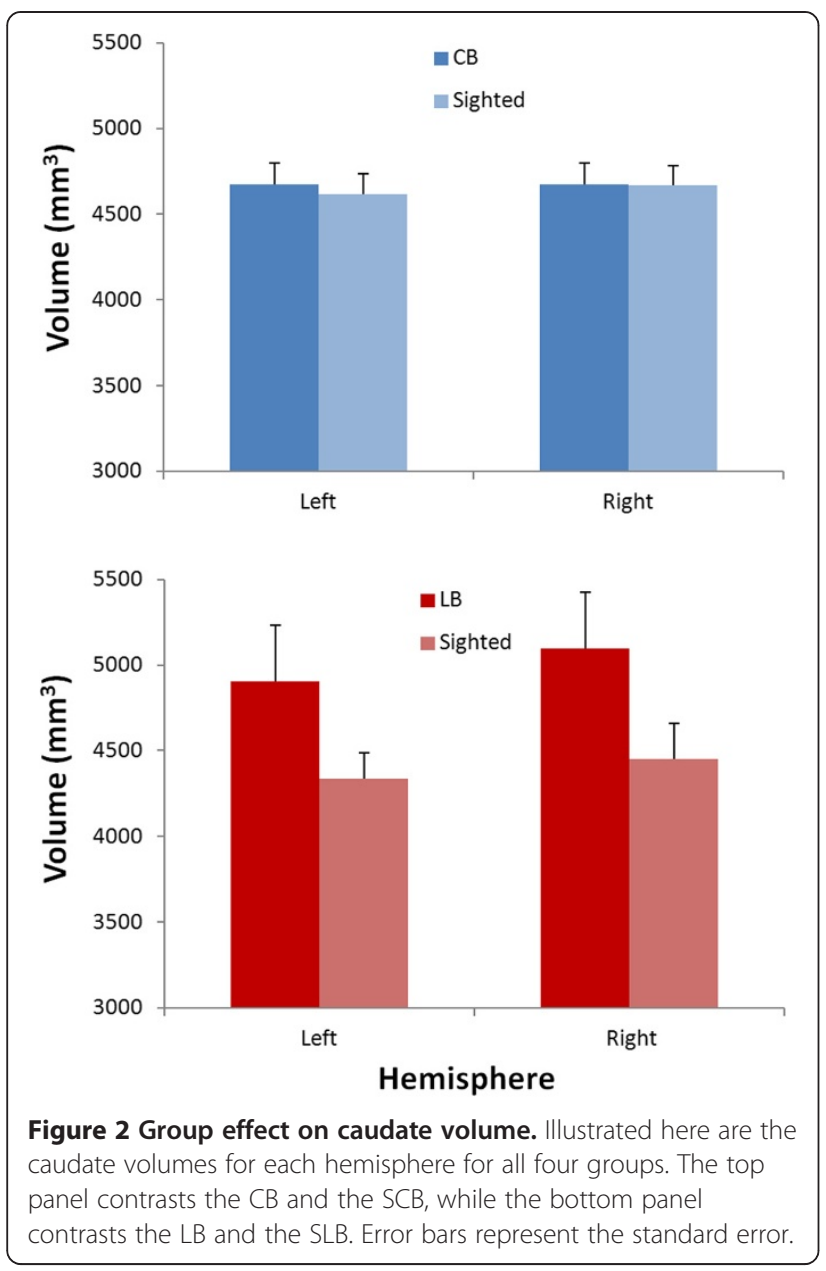

\section{Correlational analyses}

We correlated caudate volumetric measurements with several other measures to address aforementioned hypotheses. Both sighted control groups were pooled together, as there is no a priori reason to not consider them along a continuum of individuals with normal sensory experience. Regression analyses involving the blind groups were performed with both groups pooled together, as well as separately since both groups have differential experience with the visual modality.

We first ascertained whether or not caudate volume would be predictive of performance on a spatial navigation task. The task is briefly explained above, and entailed the learning of 4 different routes of increasing difficulty within a human size labyrinth. Previously, the size of the right hippocampus was shown to be predictive of performance. For a more detailed description of the task and of the findings, please see Fortin et al. [11]. As previously done, we chose to use the performance of each subject (number of errors) on the most difficult route as regressors in correlational analyses with caudate volume. However, contrary to what was observed with the hippocampus, no such correlation between performance and caudate volume was observed when correlating both measures across all participants $[\mathrm{lCN}(\mathrm{r}=0.011 ; \mathrm{p}=0.955) ; \mathrm{rCN}(\mathrm{r}=-0.144 ; \mathrm{p}=0.447)]$.

We also investigated the relationship between the total caudate volume and the total hippocampal volume previously measured in the same subjects (Fortin et al., [11]). We found no significant correlation between the volumes of both structures in any of the groups [CB $(\mathrm{r}=-0.263$; $\mathrm{p}=0.529)$, LB $(\mathrm{r}=0.078 ; \mathrm{p}=0.869)$, Sighted $(\mathrm{r}=0.242$; $\mathrm{p}=0.385)]$.

Lastly, we examined whether age at testing was related to $\mathrm{CN}$ volume. As seen in Figure 3, caudate volume was inversely correlated with age for sighted subjects $[\mathrm{ICN}$ $(\mathrm{r}=-0.737 ; \mathrm{p}=0.002) ; \mathrm{rCN}(\mathrm{r}=-0.804 ; \mathrm{p}<0.001)]$. However, this relationship was much weaker and did not reach statistical significance either for the CB $[1 C N(r=-0.388$; $\mathrm{p}=0.342) ; \mathrm{rCN}(\mathrm{r}=-0.353 ; \mathrm{p}=0.391)]$ or for the LB $[\mathrm{lCN}$ $(\mathrm{r}=-0.485 ; \mathrm{p}=0.367) ; \mathrm{rCN}(\mathrm{r}=-0.405 ; \mathrm{p}=0.367)]$. When pooling both blind groups together, given the similar correlation coefficients, the correlation coefficients became smaller and remained non-significant $[\mathrm{lCN}(\mathrm{r}=-0.331$; $\mathrm{p}=0.229) ; \mathrm{rCN}(\mathrm{r}=-0.200 ; \mathrm{p}=0.475)]$. A direct comparison of the correlation coefficients between the sighted and the blind, using Fisher's z-transform, shows that they are significantly different from one another for the right caudate $(\mathrm{z}=2.20, \mathrm{p}=0.026)$; the difference for the left caudate failed to reach statistical significance however $(\mathrm{z}=1.47, \mathrm{p}=0.142)$.

\section{Discussion}

The primary goals of the present study were: 1) to address whether or not the $\mathrm{CN}$ volume is affected in blindness, 2) to ascertain if it plays a role in the superior wayfinding abilities of blind individuals. Importantly, we also investigated the role played by the age of blindness onset on the $\mathrm{CN}$ (by separately comparing $\mathrm{CB}$ and LB to groups of sighted controls). Lastly, we also investigated whether blindness would alter associations the caudate volume has with hippocampal volume (disputed) and age.

\section{Effect of blindness}

The $\mathrm{CN}$ volume was not significantly different in the $\mathrm{CB}$ compared to matched sighted controls (SCB). However, while Figure 2 seems to indicate that the $\mathrm{CN}$ is larger in the LB compared to the SLB, this difference did not reach statistical significance, possibly due to the small subject sample and high variability. The latter contrast also revealed a significant effect of hemisphere, with the right caudate being larger than the left for each group. This hemispheric asymmetry is consistent with previous research showing that the right caudate tends to be larger than the left caudate, irrespective of gender and laterality $[28,29]$. It is however unclear to us why this asymmetry was not found for the CB-SCB contrast. 


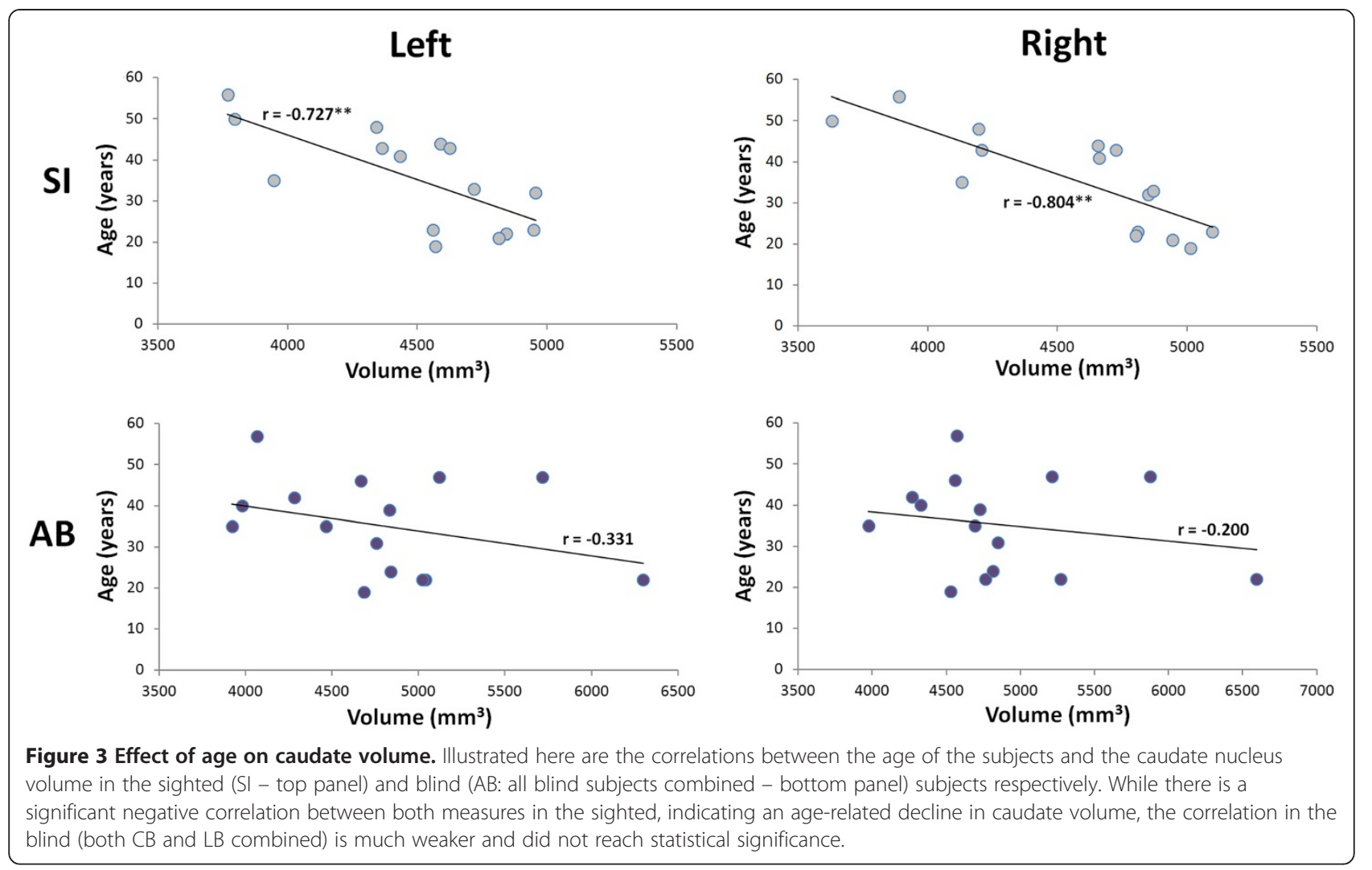

Since our LB and SLB groups are older, perhaps the CN asymmetry is exacerbated with aging. Such a hypothesis is however speculative and remains to be further explored.

We also chose to compare the $\mathrm{CN}$ measurements with those of previously obtained hippocampal measurements [11] in an attempt to address inconsistencies in the literature. Here we show that both measures are uncorrelated in all groups. This is in marked contrast to two separate lines of findings. The first stems from a previously mentioned study investigating the neural correlates of spatial navigation and showed that the size of both structures are negatively correlated with one another [9]. This inverse correlation is consistent with the authors' findings of the hippocampus being larger in 'spatial' learners and of the $\mathrm{CN}$ being larger in 'response' learners. The second line of evidence stems from work investigating the effect of aging on cortical and subcortical structures, which has shown the volume of both the hippocampus and the $\mathrm{CN}$ to correlate with one another $[19,20]$. However in the latter studies, the correlation coefficients were modest (ranging between 0.24 and 0.4 ) and likely reached statistical significance due to the large sample sizes $(>65)$. Interestingly, the correlation found here in the sighted was also of 0.24 (though with a much smaller sample size), indicating a certain level of agreement between both datasets. It remains nonetheless difficult to reconcile our findings with those of Bohbot et al. [9], where a negative correlation was found. Consequently, the currently available data does not paint a very clear picture of the relationship between caudate and hippocampal volume. As such, further investigation will be required to elucidate the nature of the volumetric relationship, should one actually exist.

The present finding of an age-related decline of caudate volume in the sighted subjects was expected and is consistent with previous reports $[17,18]$. Surprisingly however, no such relationship was found in the blind. Furthermore, the strength of the age - caudate volume relationship was shown to be significantly different between the blind and the sighted for the right caudate, suggesting that blindness does perhaps affect the caudate volume in a more subtle manner than anticipated. The absence of a significant relationship in the blind appears to, at least partly, result from the fact that the $\mathrm{CN}$ volume in the older LB individuals isn't reduced compared to the $\mathrm{CB}$ (see Figure 2). It would therefore appear that the loss of sight after puberty alters the normal developmental time-course of the age-related decline in caudate volume. The absence of an age-volume relationship in the blind could also possibly result from differential visual inputs into the tail of the $\mathrm{CN}$ via corticostriatal connections with extrastriate visual areas [30,31].

Taken together, the present findings indicate that the $\mathrm{CN}$ is not a beneficiary of compensatory plastic changes in blind individuals, as is the hippocampus $[11,13]$ and 
visually-deafferented cortical areas. Indeed, there are numerous published reports showing that occipital brain function (see [32]) and occipital neuroanatomical changes [33] appear to underlie superior abilities in a wide-range of non-visual perceptual and cognitive domains. The present null finding might be due to the CN's heterogeneous functionality which is largely motor-learning related, and for which there is little evidence of measured enhancements in the blind population. In contrast, the hippocampus' primary functions are related to memory and spatial encoding, both for which the blind have been shown to develop superior abilities in $[10,11,32]$. The visually deafferented occipital cortex however, appears to underlie a variety of enhanced perceptual and cognitive skills in the blind. As such, given that route learning is but one of many functions subtended by the $\mathrm{CN}$, it is possible that it is insufficient to drive substantial compensatory changes following visual deprivation.

\section{Relation to task performance}

Given the nature of the spatial navigation task, where a prominent strategy for success was to sequentially recall the series of right and left, we had hypothesized that the $\mathrm{CN}$ would be a likely structure to be called upon, and that its volume would be predictive of performance as previous studies have shown [9]. Unexpectedly however, the $\mathrm{CN}$ volume was not at all predictive of performance. This finding was of course unexpected given the nature of the task and the known association between the type of task used and grey matter volume in the $\mathrm{CN}$. Importantly though, it is worth pointing out that volume does not always correlate with performance in spatial navigation tasks, but rather correlates with strategies as previously underlined [9]. This was highlighted in a recent study where the hippocampal volume was found to correlate only with the use of spatial memory strategies, and not with performance on a navigation task [34]. Consequently, the absence of a correlation might simply reflect the use of differential strategies across our subject sample when performing the route learning task. However, without quantitative data on the strategies used by the participants, it is unfortunately difficult to explain the lack of a correlation with more certainty. Future work should take care in assessing the strategies used by the participants to address these unanswered questions.

\section{Conclusions}

The present data suggest that compensatory plasticity in blindness does not extend to sub-cortical structures in the striatum, as the $\mathrm{CN}$ was found to be equal in volume between sighted and blind subjects (although a statistically non-significant increase was observed in the LB relative to sighted controls), and did not correlate with performance on of the route learning task. Evidently, further investigations are required in order to better understand the role visual deprivation plays in shaping the neuroanatomy of the $\mathrm{CN}$, and how this interplays with route learning abilities.

\section{Competing interests}

The authors declare that they have no competing interests.

\section{Authors' contributions}

Conception and design: PV, MF, JCP, FL. Data collection: PV, MF. Data analysis and interpretation: PV, VC, FL. Drafting of manuscript: PV. Revising and editing of manuscript: MF, VC, JCP, FL. All authors read and approved the final manuscript.

\section{Acknowledgements}

We thank all the participants who took part in this study. This study was supported by grants from the Canadian Institutes of Health Research (CIHR) awarded to FL. PV was funded by the Natural Sciences and Engineering Research Council of Canada (NSERC).

\section{Author details}

${ }^{1}$ Montreal Neurological Institute, McGill University, Montréal, Canada. ${ }^{2}$ Centre de Recherche en Neuropsychologie et Cognition (CERNEC), Université de Montréal, Montréal, Canada. ${ }^{3}$ Douglas Hospital Research Center, McGill University, Montréal, Québec, Canada.

Received: 20 December 2012 Accepted: 18 September 2013 Published: 4 October 2013

\section{References}

1. O'Keefe J, Nadel L: The hippocampus as a cognitive Map. Oxford: Clarendon; 1978

2. McDonald RJ, White NM: Parallel information processing in the watermaze: evidence for independent memory systems involving dorsal striatum and hippocampus. Behav Neural Biol 1994, 109:579-593.

3. White NM, McDonald RJ: Multiple parallel memory systems in the brain of the rat. Neurobiol Learn Mem 2002, 77:125-184.

4. Packard MG, McGaugh JL: Inactivation of hippocampus or caudate nucleus with lidocaine differentially affects expression of place and response learning. Neurobiol Learn Mem 1996, 65:65-72.

5. Packard MG, Knowlton BJ: Learning and memory functions of the basal ganglia. Annu Rev Neurosci 2002, 25:563-593.

6. Eichenbaum H, Stewart C, Morris HG: Hippocampal representation in place learning. J Neurosci 1990, 10:3531-3542.

7. Hartley T, Maguire EA, Spiers HJ, Burgess N: The well-worn route and the path less travelled: distinct neural bases of route following and wayfinding in humans. Neuron 2003, 37:877-888.

8. laria G, Petrides M, Dagher A, Pike B, Bohbot VD: Cognitive strategies dependent on the hippocampus and caudate nucleus in human navigation: variability and change with practice. J Neurosci 2003, 23:5945-5952.

9. Bohbot VD, Lerch J, Thorndycraft B, laria G, Zijdenbos AP: Gray matter differences correlate with spontaneous strategies in a human virtual navigation task. J Neurosci 2007, 27:10078-10083.

10. Tinti C, Adenzato $M$, Tamietto $M$, Cornoldi $C$ : Visual experience is not necessary for efficient survey spatial cognition: evidence from blindness. Q J Exp Psychol 2006, 59:1306-1328.

11. Fortin M, Voss P, Lord C, Lassonde M, Pruessner J, Saint-Amour D, Rainville C, Lepore F: Wayfinding in the blind: larger hippocampal volume and supranormal spatial navigation. Brain 2008, 131:2995-3005.

12. Chebat DR, Chen JK, Schneider F, Ptito A, Kupers R, Ptito M: Alterations in right posterior hippocampus in early blind individuals. NeuroReport 2007 18:329-333.

13. Lepore $N$, Shi Y, Lepore F, Fortin M, Voss P, Chou Y, Lord C, Lassonde M, Dinov I, Toga AW, Thompson PM: Patterns of hippocampal shape and volume changes in blind subjects. Neuroimage 2009, 46:949-957.

14. Raz N, Striem E, Pundak G, Orlov T, Zohary E: Superior serial memory in the blind: a case of cognitive compensatory adjustment. Curr Biol 2007, 17:1129-1133 
15. Iverson JM: How to get to the cafeteria: gesture and speech in blind and sighted children's spatial descriptions. Dev Psychol 1999, 35:1131-1142.

16. Head D, Isom M: Age effects on wayfinding and route learning skills. Behav Brain Res 2010, 209:49-58.

17. Hasan KM, Halphen C, Kamali A, Nelson FM, Wolinsky JS, Narayana PA: Caudate nuclei volume, diffusion tensor metrics, and $\mathrm{T}(2)$ relaxation in healthy adults and relapsing-remitting multiple sclerosis patients: implications for understanding gray matter degeneration. J Magn Reson Imaging 2009, 29:70-77.

18. Walhovd KB, Fjell AM, Reinvang I, Lundervold A, Dale AM, Eilertsen DE, Quinn BT, Salat D, Makris N, Fischl B: Effects of age on volumes of cortex, white matter and subcortical structures. Neurobiol Aging 2005, 26:1260-1270.

19. Raz N, Williamson A, Gunning-Dixon F, Head D, Acker JD: Neuroanatomical and cognitive correlates of adult age differences in acquisition of a perceptual-motor skill. Microsc Res Tech 2000, 51:85-93.

20. Raz N, Lindenberger U, Rodrigue KM, Kennedy KM, Head D, Williamson A, Dahle C, Gerstorf D, Acker JD: Regional brain changes in aging healthy adults: general trends, individual differences and modifiers. Cereb Cortex 2005, 15:1676-1689.

21. Oldfield RC: The assessment and analysis of handedness: the Edinburgh inventory. Neuropsychologia 1971, 9:97-113.

22. Sled JG, Zijdenbos AP, Evans AC: A nonparametric method for automatic correction of intensity nonuniformity in MRI data. IEEE Trans Med Imaging 1998, 17:87-97.

23. Collins DL, Neelin P, Peters TM, Evans AC: Automatic 3D intersubject registration of MR volumetric data in standardized talairach space. J Comput Assist Tomogr 1994, 18:192-205.

24. Collins DL, Zijdenbos AP, Kollokian V, Sled JG, Kabani NJ, Holmes CJ, et al: Design and construction of a realistic digital brain phantom. IEEE Trans Med Imaging 1998, 17:463-468.

25. Collins DL, Evans AC: ANIMAL: Validation and applications of nonlinear registration-based segmentation. Intern J Pattern Recognit Artif Intell 1997 11:1271-1294

26. Pruessner JC, Li LM, Serles W, Pruessner M, Collins DL, Kabani N, Lupien S, Evans AC: Volumetry of hippocampus and amygdala with high-resolution $\mathrm{MRI}$ and threedimensional analysis software: minimizing the discrepancies between laboratories. Cereb Cortex 2000, 10:433-442.

27. Pruessner JC, Collins DL, Pruessner M, Evans AC: Age and gender predict volume decline in the anterior and posterior hippocampus in early adulthood. J Neurosci 2001, 21:194-200

28. Ifthikharuddin SF, Shrier DA, Numaguchi Y, Tang X, Ning R, Shibata DK Kurlan R: MR volumetric analysis of the human basal ganglia: normative data. Acad Radiol 2000, 7:627-634.

29. Peterson BS, Riddle MA, Cohen DJ, Katz LD, Smith JC, Leckman JF: Human basal ganglia volume asymmetries on magnetic resonance images. Magn Reson Imaging 1993, 11:493-498.

30. Selemon LD, Goldman-Rakic PS: Longitudinal topography and interdigitation of corticostriatal projections in the rhesus monkey. J Neurosci 1985, 5:776-794.

31. Yeterian EH, Pandya DN: Corticostriatal connections of extrastriate visual areas in rhesus monkeys. J Compar Neurol 1995, 352:436-457.

32. Voss $\mathrm{P}$, Collignon $\mathrm{O}$, Lassonde M, Lepore F: Adaptation to sensory loss. WIREs Cog Sci 2010, 1:308-328.

33. Voss $P$, Zatorre R: Occipital cortical thickness predicts performance on pitch and musical tasks in blind individuals. Cereb Cortex 2012, 22:2455-2465.

34. Konishi K, Bohbot VD: Spatial navigation strategies correlate with gray matter in the hippocampus of healthy older adults tested in a virtual maze. Front Aging Neurosci 2013, 5:1-8.

doi:10.1186/1471-2202-14-113

Cite this article as: Voss et al: Assessment of the caudate nucleus and its relation to route learning in both congenital and late blind individuals. BMC Neuroscience 2013 14:113.

\section{Submit your next manuscript to BioMed Central and take full advantage of:}

- Convenient online submission

- Thorough peer review

- No space constraints or color figure charges

- Immediate publication on acceptance

- Inclusion in PubMed, CAS, Scopus and Google Scholar

- Research which is freely available for redistribution

Submit your manuscript at www.biomedcentral.com/submit
C Biomed Central 